PROCEEDINGS OF THE

AMERICAN MATHEMATICAL SOCIETY

Volume 130, Number 6, Pages 1623-1627

S 0002-9939(01)06461-9

Article electronically published on November 15, 2001

\title{
SIGNED SUMS OF POLYNOMIAL VALUES
}

\author{
HONG BING YU \\ (Communicated by David E. Rohrlich)
}

\begin{abstract}
We give a generalization of Bleicher's result on signed sums of $k$ th powers. Let $f(x)$ be an integral-valued polynomial of degree $k$ satisfying the necessary condition that there exists no integer $d>1$ dividing the values $f(x)$ for all integers $x$. Then, for every positive integer $n$ and every integer $l$, there are infinitely many integers $m \geq l$ and choices of $\varepsilon_{i}= \pm 1$ such that
\end{abstract}

$$
n=\sum_{i=l}^{m} \varepsilon_{i} f(i) \text {. }
$$

In [1] Bleicher proved, among other things, the following interesting result: every positive integer $n$ can be written in the form

$$
n=\sum_{i=1}^{m} \varepsilon_{i} i^{k} \quad \text { with suitable } \varepsilon_{i}= \pm 1
$$

where $k \geq 2$ is any given integer, and $m \geq 1$ is an integer, depending on $n$ and $k$. The purpose of this note is to consider an analogous problem for polynomials (cf. [2], 3], [5] Chap.12]). We shall prove:

Theorem. Let $f(x)$ be an integral-valued polynomial of degree $k$ satisfying the necessary condition that there exists no integer $d>1$ dividing the values $f(x)$ for all integers $x$. Then, for any given integer $l$, every positive integer $n$ can be represented in the form

$$
n=\sum_{i=l}^{m} \varepsilon_{i} f(i) \quad \text { with suitable } \varepsilon_{i}= \pm 1,
$$

where $m \geq l$ is an integer, depending on $l, n$ and $f(x)$.

For the proof of the theorem we begin with some preliminaries. It is well-known (cf. [2]) that the condition of the theorem is equivalent to $f(x)$ being of the form

$$
f(x)=a_{k}\left(\begin{array}{l}
x \\
k
\end{array}\right)+\cdots+a_{1}\left(\begin{array}{l}
x \\
1
\end{array}\right)+a_{0},
$$

where $\left(\begin{array}{l}x \\ i\end{array}\right)=\frac{x(x-1) \cdots(x-i+1)}{i !}(1 \leq i \leq k)$, and $a_{0}, a_{1}, \cdots, a_{k}$ are integers satisfying

$$
a_{k} \neq 0 \text { and }\left(a_{0}, a_{1}, \cdots, a_{k}\right)=1 \text {. }
$$

Our starting-point is the following two results.

Received by the editors January 10, 2001.

2000 Mathematics Subject Classification. Primary 11A67, 11P05.

The author was supported by the National Natural Science Foundation of China. 
Lemma 1. There exists a partition of $\left\{0,1, \cdots, 2^{k}-1\right\}$ as a disjoint union of two sets $A$ and $B$, such that the polynomial identity

$$
\sum_{a \in A} f(x+a)-\sum_{b \in B} f(x+b)=2^{\frac{k(k-1)}{2}} a_{k}
$$

holds.

Proof. This result is related to the Tarry-Escott problem. It is known that, for each positive integer $h$, there is a partition of $\left\{0,1, \cdots, 2^{h}-1\right\}$ into sets $A, B$ with $|A|=|B|$, such that

$$
\sum_{a \in A} a^{j}-\sum_{b \in B} b^{j}=0 \text { for } j=1, \cdots, h-1,
$$

and

$$
\sum_{a \in A} a^{h}-\sum_{b \in B} b^{h}=h ! 2^{\frac{h(h-1)}{2}} .
$$

(For a simple proof of this fact see [4]; cf. also [1, §2].)

Let $f(x)=\alpha_{k} x^{k}+\cdots+\alpha_{1} x+\alpha_{0}$; then $\alpha_{k}=\frac{a_{k}}{k !}$. By (5), (6) (with $h=k$ ) and the binomial theorem we infer that the left side of (4) equals $k ! 2^{\frac{k(k-1)}{2}} \alpha_{k}=2^{\frac{k(k-1)}{2}} a_{k}$, as stated.

Lemma 2. Write $M=2^{\frac{k(k-1)}{2}} a_{k}$. If for a positive integer $n$ there exists an integer $s \geq l$ such that

$$
\sum_{i=l}^{s} \varepsilon_{i}^{\prime} f(i) \equiv n(\bmod M) \quad \text { with suitable } \varepsilon_{i}^{\prime}= \pm 1
$$

then the integer $n$ is representable in the form (1) for a certain $m \geq l$.

Proof. We denote by $g(x)$ the polynomial of the left side of (4). Then, for any integer $u, g(u)$ is a signed sum of the values of $f(x)$ at consecutive integers $u, u+$ $1, \cdots, u+2^{k}-1$. The assumptions imply that for a certain integer $L$ we have

$$
\sum_{i=l}^{s} \varepsilon_{i}^{\prime} f(i)=n+L M
$$

Thus by Lemma 1 ,

$$
n=\sum_{i=l}^{s} \varepsilon_{i}^{\prime} f(i)-(\operatorname{sign} L) \sum_{t=0}^{|L|-1} g\left(s+t \cdot 2^{k}+1\right),
$$

and this gives the desired representation of $n$ (cf. [1, §3]).

Thus to prove the theorem it suffices to establish the solubility of (7) for every integer $n \geq 1$. For this purpose we need two more lemmas.

Lemma 3. Let $l$ be any given integer. Then $(f(l), f(l+1), \cdots, f(l+k))=1$.

Proof. Let $d=(f(l), f(l+1), \cdots, f(l+k))$, and let $h(x)=\frac{f(x)}{d}$. Then $\operatorname{deg} h=k$ and the values of $h(x)$ at $k+1$ consecutive integers $l, l+1, \cdots, l+k$ are integers. Thus $h(x)$ is an integral-valued polynomial. In particular, $h(0), h(1), \cdots, h(k)$ are all integers. It follows from (2) that $d \mid a_{i}(i=0,1, \cdots, k)$. Thus $d=1$ by (3). 
Lemma 4. Let $b_{1}, \cdots, b_{k}$ be integers, not all zero, and let $d=\left(b_{1}, \cdots, b_{k}\right)$. If $d$ is an odd number, then there exist odd $x_{1}, \cdots, x_{r}$ and even $x_{r+1}, \cdots, x_{k}(1 \leq r \leq k)$ such that

$$
b_{1} x_{1}+\cdots+b_{k} x_{k}=d .
$$

Proof. We proceed by induction on $k$. When $k=2$ the result is trivial. In fact, it is easily verified that if $b_{1}$ is odd, we can choose $x_{1}$ odd and $x_{2}$ even; if $b_{1}$ is even, we can choose $x_{1}$ and $x_{2}$ both odd.

Suppose that $k \geq 3$ and that result is true with $k$ replaced by $k-1$. Clearly, we may assume that $b_{2}, \cdots, b_{k}$ are not all zero, and let $d_{1}=\left(b_{2}, \cdots, b_{k}\right)$. If $b_{1}$ is odd, since $\left(b_{1}, d_{1}\right)=d$ is odd, it follows that there exist odd $y_{1}$ and even $y_{2}$ such that $b_{1} y_{1}+d_{1} y_{2}=d$. Moreover, there are integers $x_{2}^{\prime}, \cdots, x_{k}^{\prime}$ such that $\sum_{i=2}^{k} x_{i}^{\prime} b_{i}=d_{1}$. Thus

$$
b_{1} y_{1}+\sum_{i=2}^{k}\left(y_{2} x_{i}^{\prime}\right) b_{i}=d \text {, }
$$

as required.

If $b_{1}$ is even, then $d_{1}$ must be odd. By the induction hypothesis, there are odd $x_{2}^{\prime}, \cdots, x_{v}^{\prime}$ and even $x_{v+1}^{\prime}, \cdots, x_{k}^{\prime}(2 \leq v \leq k)$ such that $\sum_{i=2}^{k} x_{i}^{\prime} b_{i}=d_{1}$. Furthermore, since $b_{1}$ is even, there exist $y_{1}$ and $y_{2}$, both odd, satisfying $b_{1} y_{1}+d_{1} y_{2}=d$. Thus

$$
b_{1} y_{1}+\sum_{i=2}^{k}\left(y_{2} x_{i}^{\prime}\right) b_{i}=d
$$

and this gives the desired result.

Now we prove that (7) is soluble for every integer $n \geq 1$, and thus complete the proof of the theorem. Let $q>0$ be the least common denominator of the coefficients of $f(x)$. Write $M^{*}=q|M|$. Then $k \leq M^{*}$ and

$$
f\left(u M^{*}+i\right) \equiv f(i)(\bmod M) \text { for any integers } u \text { and } i .
$$

Moreover, by Lemmas 2 and 3 , there exist odd $x_{0}, \cdots, x_{r}$ and even $x_{r+1}, \cdots$, $x_{k}(0 \leq r \leq k)$ such that

$$
\sum_{i=0}^{k} x_{i} f(i+l)=1 .
$$

In the following we fix such integers $x_{0}, \cdots, x_{k}$, and put

$$
\eta_{i}=\operatorname{sign} x_{i} \quad(i=0, \cdots, k) .
$$

We distinguish two cases.

(i) $n$ is even. Let $L_{-1}=0$ and $L_{i}=\frac{n}{2}\left(\left|x_{0}\right|+\cdots+\left|x_{i}\right|\right)(i=0, \cdots, k)$. By $(8)$ we have, for any integers $u$ and $0 \leq i \leq k$,

$$
\begin{aligned}
& \sum_{\substack{j=0 \\
j \neq i}}^{M^{*}-1} f\left(2 u M^{*}+l+j\right)-\sum_{\substack{j=0 \\
j \neq i}}^{M^{*}-1} f\left((2 u+1) M^{*}+l+j\right) \\
& \quad+\eta_{i} f\left(2 u M^{*}+l+i\right)+\eta_{i} f\left((2 u+1) M^{*}+l+i\right) \\
& \equiv 2 \eta_{i} f(l+i)(\bmod M) .
\end{aligned}
$$


Now, summing for $u$ from $L_{i-1}$ to $L_{i}$, we see that there exist $\varepsilon_{j}^{\prime}= \pm 1$ such that

$$
\sum_{j=2 L_{i-1} M^{*}+l}^{2 L_{i} M^{*}+l-1} \varepsilon_{j}^{\prime} f(j) \equiv 2 \cdot \frac{n}{2}\left|x_{i}\right| \eta_{i} f(l+i) \equiv n x_{i} f(l+i)(\bmod M) .
$$

Summing for $i=0,1, \cdots, k$ and using (9), we obtain the desired result:

$$
\sum_{j=l}^{2 L_{k} M^{*}+l-1} \varepsilon_{j}^{\prime} f(j) \equiv n \sum_{i=0}^{k} x_{i} f(l+i)=n(\bmod M)
$$

(ii) $n$ is odd. We define $\delta_{i}=\frac{n\left|x_{i}\right|-1}{2}$ for $0 \leq i \leq r$ and $\delta_{i}=\frac{n\left|x_{i}\right|}{2}$ for $r+1 \leq i \leq k$. Then all $\delta_{i}$ are integers. Further, let $L_{-1}=0$ and $L_{i}=\delta_{0}+\cdots+\delta_{i}(i=0, \cdots, k)$. In analogy to $(11)$, there exist $\varepsilon_{j}^{\prime}= \pm 1$ such that

$$
\sum_{j=2 L_{i-1} M^{*}+l}^{2 L_{i} M^{*}+l-1} \varepsilon_{j}^{\prime} f(j) \equiv n x_{i} f(l+i)-\eta_{i} f(l+i)(\bmod M)
$$

for $0 \leq i \leq r ;$ and

$$
\sum_{j=2 L_{i-1} M^{*}+l}^{2 L_{i} M^{*}+l-1} \varepsilon_{j}^{\prime} f(j) \equiv n x_{i} f(l+i)(\bmod M)
$$

for $r+1 \leq i \leq k$. Thus

$$
\sum_{j=l}^{2 L_{k} M^{*}+l-1} \varepsilon_{j}^{\prime} f(j) \equiv n-\sum_{i=0}^{r} \eta_{i} f(l+i)(\bmod M) .
$$

Finally, by (8) we have

$$
\sum_{i=0}^{r} \eta_{i} f\left(2 L_{k} M^{*}+l+i\right) \equiv \sum_{i=0}^{r} \eta_{i} f(l+i)(\bmod M)
$$

The desired result follows from (10), (12) and (13). The proof of the theorem is now complete.

Remarks. 1. By the result cited in the proof of Lemma 1, it is easy to see that, for any integers $h>k$ and $x$, there exist $\varepsilon_{i}= \pm 1$ such that

$$
\sum_{i=x}^{x+2^{h}-1} \varepsilon_{i} f(i)=0 .
$$

It follows that, for every integer $n \neq 0$, there are infinitely many $m$ such that $(1)$ holds (cf. the proof of Corollary 2 in 1]). Moreover, (14) (with $x=l$ ) shows that for $n=0$ a representation of the form (1) still exists; in fact, infinitely many exist.

2. When $f(x)$ and $l$ are fixed and $n$ grows to infinity, it seems to be more difficult to estimate the minimal value of $m$ in (1). For the case $f(x)=x^{k}$, this has been done by Bleicher in [1, §4]. 


\section{REFERENCES}

[1] M. N. Bleicher, On Prielipp's problem on signed sums of $k$ th powers, J. Number Theorey. 56(1996), 36-51. MR 96j:11011

[2] R. L. Graham, Complete sequences of polynomial values, Duke Math.J, 31(1964), 275-285. MR 29:63

[3] L. K. Hua, An easier Waring-Kamke problem, J. London Math. Soc. 11(1936), 4-5.

[4] D. E. Knuth and José Heber Nieto, Solution to Problem E3303, Amer. Math. Monthly. 97(1990), 348-349.

[5] M. B. Nathanson, "Elementary Methods in Number Theory", volume 195 of Graduate Texts in Mathematics, Springer-Verlag, 2000. MR 2001j:11001

Department of Mathematics, University of Science and Technology of China, Hefei 230026, Anhui, People's Republic of China

E-mail address: yuhb@ustc.edu.cn 